\title{
Density-functional theory calculation of electronic and magnetic properties of hexagonal V/Ru thin films and superlattices
}

\author{
Tianjiao Zhang* \\ Department of Physics, National Taiwan University, Taipei, Taiwan 106, Republic of China \\ G. Y. Guo ${ }^{\dagger}$ \\ Department of Physics, National Taiwan University, Taipei, Taiwan 106, Republic of China \\ and National Synchrotron Radiation Research Center, Hsinchu, Taiwan 300, Republic of China \\ (Received 5 October 2004; revised manuscript received 28 January 2005; published 30 June 2005)
}

\begin{abstract}
Stimulated by recent experimental evidence for the formation of ultrathin close-packed films of vanadium (V) and also indication of ferromagnetic order in epitaxial $\mathrm{V} / \mathrm{Ru}$ multilayers, we have performed firstprinciples electronic structure and total energy calculations to study the stability and magnetism of the metastable hcp and fcc structures of $\mathrm{V}$ and also the effects of the surface and interface on these properties. The systems include ultrathin films of a few monolayers of V grown on hcp (0001) ruthenium (Ru) substrates and $\mathrm{V} / \mathrm{Ru}$ superlattices as well as bulk bcc, fcc, and hep structures. Our investigations are based on densityfunctional theory with local density approximation plus generalized gradient corrections. Both the frozen-core projector augmented-wave approach and the all-electron full-potential linearized augmented-plane-wave method are utilized. First, our calculations show that all three bulk structures are nonmagnetic at their respective minimal energy lattice constants. However, all the three structures transit first to a low-spin ferromagnetic phase and then to a high-spin phase as the lattices expand. Second, we find that thin films on hcp (0001) Ru with one monolayer of $\mathrm{V}$ in either hcp or fcc stacking sequence have a magnetic moment of the order of $1 \mu_{B}$. A thin film of three monolayers of $\mathrm{V}$ in fcc stacking sequence is weakly ferromagnetic, though the other thin films with more than one monolayer of $\mathrm{V}$ are essentially nonmagnetic. A free-standing film of three $\mathrm{V}$ monolayers in hep stacking sequence is also ferromagnetic with sizable magnetic moments. We also find that the $\mathrm{V}_{2}(\mathrm{hcp}) / \mathrm{Ru}_{6}(\mathrm{hcp})$ and $\mathrm{V}_{3}(\mathrm{hcp}) / \mathrm{Ru}_{5}(\mathrm{hcp})$ superlattices exhibit ferromagnetism with a small total magnetic moment of a few tenths of $\mu_{B}$. Lattice relaxation has the trend of decreasing the magnitude of the magnetic moments. All the other $\mathrm{V}_{n} / \mathrm{Ru}_{m}$ (hcp) superlattices $(n=1,4,5$ and $m=5,6$ as well as $n=3$ with fcc or bcc stacking sequence) are essentially nonmagnetic. Finally, our calculations show that the stacking sequence has significant effects on the formation of stable atomic magnetic moments of $\mathrm{V}$ and is also important for the energetic stability of these systems.
\end{abstract}

DOI: 10.1103/PhysRevB.71.214442

PACS number(s): 75.70.-i, 61.66.Bi, 62.20.Dc, 71.15.Nc

\section{INTRODUCTION}

Recent progress in molecular beam epitaxy growth techniques and a better understanding, monitoring, and controlling of growth conditions have made it possible to fabricate many artificial thin films and superlattice structures (see, Refs. 1-4 and references therein). Electrons in these atomic systems usually encounter interactions different from what they would encounter in natural crystals. This effect has provided us more opportunities to explore novel physical properties, particularly in the pursuit of magnetic materials. Despite the fact that many $3 d$ and $4 d$ transition-metal atoms have magnetic moments, only $\mathrm{Fe}, \mathrm{Co}, \mathrm{Ni}, \mathrm{Cr}$, and $\mathrm{Mn}$ are magnetic when they form crystal structures. Therefore, scientists are very interested in building artificial film structures or metastable phases with these five elements. Well-known examples include fcc Fe on $\mathrm{Cu},{ }^{1}$ bcc $\mathrm{Co}$ on $\mathrm{GaAs},{ }^{2}$ bcc $\mathrm{Ni}$ on $\mathrm{Cu},{ }^{5}$ hep $\mathrm{Cr}$ on $\mathrm{Ru},{ }^{6}$ and $\mathrm{Mn}$ on GaAs. ${ }^{7}$ However, stabilization of magnetic phases in structures formed with other nonmagnetic transition metals is less promising. In this context, vanadium is a hot candicate because of its large magnetic moment in free atomic form. And there are quite a few studies of systems associated with V (see, e.g., Refs. 8 and 9 and references therein). In general, the more free atom like the $\mathrm{V}$ atom's chemical environment is, the larger is the magnetic moment. A free $\mathrm{V}$ atom has an electron configuration $[\mathrm{Ar}] 4 s^{1} 3 d^{4}$ and a permanent spin magnetic moment of $5 \mu_{B}$. The magnetic moment of vanadium clusters varies with cluster size, on average oscillating about $0.3 \mu_{B}$ per atom for clusters containing fewer than 20 atoms (see Ref. 10 and references therein).

It is well known that $\mathrm{V}$ atoms crystallize in the bcc structure and that the material is paramagnetic. The magnetic moment on the bcc V surfaces varies with surface orientation. Surfaces such as bcc (111) have small magnetic moments of about $0.05 \mu_{B}$ while close-packed surfaces such as bcc (110) are nonmagnetic even when expanded up to $7 \%$ of the surface lattice constant. ${ }^{11}$ The bcc (100) surface is a structure more dense than bcc (111) but less dense than bcc (110). Thus, a conclusive determination of magnetic properties of this surface structure is more difficult to achieve. Fullpotential calculations with surface relaxation indicated that the bcc (100) surface of $\mathrm{V}$ is nonmagnetic, which is confirmed in Ref. 11 using surface Green function method as well. Pseudopotential calculations need to be extremely careful as they can otherwise give false magnetic moments as 
large as $0.75 \mu_{B} \cdot{ }^{11-13}$ In Ref. 12 , it is reported that the bcc (100) $\mathrm{V}$ surface is magnetic with a magnetic moment of $0.75 \mu_{B}$ from pseudopotential calculations while in contrast it is nonmagnetic from all-electron full-potential calculations. As explained later in Ref. 13, this apparent controversy could be resolved by using a more accurate representation of $d$ wave functions in pseudopotential construction. Other studies of magnetic properties related to vanadium atoms including heterostructures such as RuV, RhV, PdV (Ref. 9), and MoV (Ref. 8) binary alloys, V/Fe (Refs. 14 and 15), Ag/V (Ref. 16), and $\mathrm{V} / \mathrm{Cu}(001)$ (Ref. 17) surface structures are reported in the literature. Our following study of the $\mathrm{V} / \mathrm{Ru}$ system is partially motivated by recent experimental evidence for the formation of ultrathin close-packed fcc or hcp $\mathrm{V}$ films in epitaxial V/Ru multilayers. ${ }^{18}$ In this system, structural stabilities similar to $\mathrm{Cr} / \mathrm{Ru}$ (0001) thin films and superlattices $^{6}$ were observed. When the thickness of $\mathrm{V}$ is below three monolayers, $\mathrm{V}$ atoms adopt the hexagonal symmetry of the $\mathrm{Ru}(0001)$ substrate and show some indication of a ferromagnetic order with a small magnetic moment. When the V layers get thicker, V atoms spontaneously switch to the bcc structure revealing no magnetic moment. ${ }^{18}$

The purpose of the present study is primarily to investigate theoretically the possible formation of stable magnetic moments of $\mathrm{V}$ in the close-packed structures of bulk formats or ultrathin multilayers. Therefore, in this paper, we report on systematic density functional calculations of thin $\mathrm{V}$ films on hcp (0001) Ru and also superlattice structures formed of $\mathrm{V}$ and $\mathrm{Ru}$ atomic layer stackings perpendicular to the hcp (0001) surface. The variation of the atomic magnetic moment of $\mathrm{V}$ with the thickness of $\mathrm{V}$ layers and the effects of the bcc, fcc, or hcp stacking sequences are explicitly explored. We also report on the calculated magnetic properties of $\mathrm{V}$ in bulk fcc and hcp as well as bcc structures.

This paper is organized as follows. In the next section, we describe briefly the methodologies and computational details. In Sec. III bulk calculation results are presented for bcc, fcc, and hcp V as well as hcp Ru. In Sec. IV, we present the results of projector augmented-wave ${ }^{19}$ (PAW) calculations for thin-film structures. In Sec. V, we report on the results of PAW and full-potential linearized augmented-plane-wave ${ }^{20}$ (FLAPW) calculations for superlattices. Some detailed analysis of the effects of lattice relaxation on the magnetic moment is also presented. Both Secs. IV and V include studies of the effects of the stacking sequence on the total energy and magnetic moment. Finally, a short summary is given in Sec. VI.

\section{METHODOLOGIES}

In our following calculations, both the PAW (Ref. 19) and FLAPW (Ref. 20) methods have been utilized. Since PAW calculations are much less CPU time consuming, FLAPW calculations are only performed in cases where we think extra care is needed to make sure that we draw conclusions based on accurate numerical results. For the PAW method, the Vienna $a b$ initio simulation package ${ }^{21}$ (VASP) is used. Atomic core electron states $3 p^{6}$ of $\mathrm{V}$ and $4 p^{6}$ of $\mathrm{Ru}$ are treated as valence electrons in the PAW potential construc- tion. A plane-wave energy cutoff of $300 \mathrm{eV}$ is used. For the FLAPW method, the WIEN2K package ${ }^{20}$ is used. The muffintin radius used for both $\mathrm{V}$ and $\mathrm{Ru}$ is 2.3 atomic units (a.u.). The atomic core states $3 s^{2} 3 p^{6}$ of $\mathrm{V}$ and $4 s^{2} 4 p^{6}$ of $\mathrm{Ru}$ are treated as valence states using the so-called local orbitals. ${ }^{20}$ Inside the muffin-tin spheres, the wave functions, charge densities, and potential are expanded in terms of the spherical harmonics. The cutoff angular momentum $\left(L_{\max }\right)$ is 10 for the wave functions and 6 for charge densities (potentials). The number of augmented plane waves included is about 90 per atom, i.e., $R_{m t} K_{\max }=9 .{ }^{20}$ It is well known that densityfunctional theory (DFT) with the standard local density approximation (LDA) does not accurately describe the properties of $3 d$ transition metals. ${ }^{22,23}$ In particular, DFT-LDA gives a wrong ground state for Fe. ${ }^{22,24}$ Nevertheless, these errors can largely be removed by including generalized gradient (GGA) corrections (see, e.g., Refs. 25-27 and references therein). Therefore, we have included the GGA of Perdew, Burke, and Ernzerhof ${ }^{26}$ in all the presented calculations. Scalar relativity effects have been included but spin-orbit coupling is not considered in these calculations. Most results are obtained by choosing an initial ferromagnetic arrangement of the electron spin orientation in each atom. For certain cases antiferromagntic calculations are also performed but the selfconsistent calculation results are not different from calculations based on ferromagnetic arrangement of the initial spin orientation. Except for pursuing the ground states of free atoms, the improved tetrahedron method ${ }^{28}$ is used to carry out Brillouin zone integration. As the number of $k$ points in the irreducible Brillouin zone wedge (IBZW) varies with different systems, it is given in each section below.

\section{BULK PROPERTIES}

Bulk calculations for $\mathrm{V}$ and $\mathrm{Ru}$ are performed with the PAW method. For V, we have performed total energy calculations to determine its bulk lattice constants for the bcc, fcc, and hcp phases. For Ru, only the hcp phase is treated. The numbers of the $k$ points in the IBZW used for bcc V, fcc V, hcp V, and hcp Ru are 145, 145, 270, and 270, respectively. The self-consistent cycles are terminated when the total energy change is smaller than $10^{-5} \mathrm{eV}$.

The equilibrium lattice constants of these bulk systems are obtained from the minimization of total energy. The results are given in Fig. 1. The values of these equilibrium lattice constants are listed in Table I and they agree well with previous studies. ${ }^{12,13,29,30}$ In Table I, we also present the nearest-neighbor distances $\left(d_{n n}\right)$ and atomic volume $(\Omega)$ of these systems. For hcp V, we list values for two different configurations. The structure with $a=2.60 \AA$ and $c / a=1.82$ is the one with the lowest total energy. The structure (the system "hcp $\mathrm{V}^{*}$ " in Table I) with $a=2.74 \AA$ and $c / a=1.65$ corresponds to the most stable structure for hcp V when it is forced to adopt the in-plane lattice constant of hcp Ru. The energy difference between these two configurations is $0.053 \mathrm{eV}$ (or about $600 \mathrm{~K}$ ). For all phases of $\mathrm{V}, d_{n n}$ is smaller than the hcp Ru (0001) in-plane lattice constant of $a$ $=2.74 \AA$. However, the difference is at most $-3 \%$. This makes it possible to have pseudomorphic growth of $\mathrm{V}$ atomic 

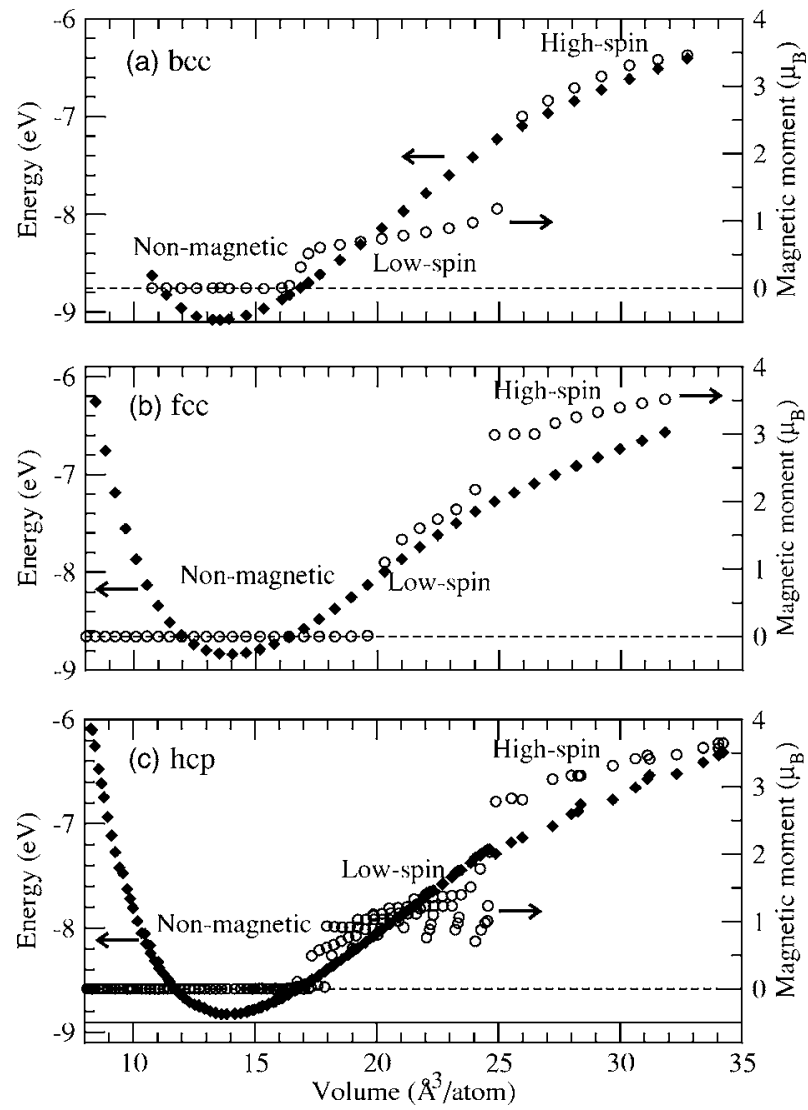

FIG. 1. Total energy (solid diamonds) and magnetic moment (open circles) of $\mathrm{V}$ as a function of atomic volume for bulk bec (a), fcc (b), and hcp (c) structures.

layers on the Ru hcp (0001) surface without surface reconstruction.

In Table I, we also list the cohesive energies for these systems. To achieve this, we have performed calculations to determine the ground states of the two atoms. For V, a bcc unit cell with a large lattice constant of $12 \AA$ is used. We have considered two methods to treat the gound-state occupation. In the first method, ${ }^{21}$ Gaussian smearing with a width of $0.08 \mathrm{eV}$ is used. A symmetry-broken spin-polarized calculation is performed. At the $\Gamma$ point, the six lowest states above the $[\mathrm{Ar}] 3 p^{6}$ have the same spin orientation and the eigenvalues are $-4.105,-3.021,-3.019,-3.016,-2.695$, and
$-2.694 \mathrm{eV}$. The first one corresponds to the $s$ orbital and the other five states are the $d$ orbitals. The finite gap between the $e_{g}$ and $t_{2 g}$ states indicates that there is still a finite crystal field which we have attempted to remove by using a large unit cell. The occupation numbers for the last two states are about 0.505 and 0.495 , respectively. The ground state obtained is about $3.753 \mathrm{eV}$ lower than the reference state used in the PAW potential construction for $\mathrm{V}$, which gives a cohesive energy of $5.328 \mathrm{eV}$ for bcc V. Explicitly considering Vosko-Wilk-Nusair ${ }^{31}$ interpolation for the correlation part of exchange correlation functional gives a $0.002-\mathrm{eV}$ lower state. In the other method, we simply used all the same parameters adopted for bulk calculation except that a larger lattice constant of $12 \AA$ was applied. The tetrahedron method is used for Brillioun zone integration. At the $\Gamma$ point, the six lowest states above the $[\mathrm{Ar}] 3 p^{6}$ also have the same spin state and the eigenvalues are $-4.103,-2.995,-2.995,-2.799,-2.799$, and $-2.799 \mathrm{eV}$. Here we do not consider explicitly Vosko-Wilk-Nusair ${ }^{34}$ interpolation (as in all our bulk, thinfilm, and superlattice calculations). The ground state for $\mathrm{V}$ determined in this way is about $3.682 \mathrm{eV}$ lower than the reference state, which gives a cohesive energy of $5.399 \mathrm{eV}$ for bcc V. Calculation with the FLAPW method gives a cohesive energy of $5.600 \mathrm{eV}$. All these methods show that the ground-state electronic structure for $\mathrm{V}$ is $[\mathrm{Ar}] 4 s^{1} 3 d^{4}$ and the magnetic moment of the free atom is $5 \mu_{B}$. The cohesive energy is close to the value of $5.31 \mathrm{eV}$ given in Ref. 32 . However, the ground state given there is $[\mathrm{Ar}] 4 s^{2} 3 d^{3}$, which is $0.870 \mathrm{eV}$ higher above the ground state in our calculation. The spin state in $[\mathrm{Ar}] 4 s^{0} 3 d^{5}$ is even $0.910 \mathrm{eV}$ higher than for $[\mathrm{Ar}] 4 s^{2} 3 d^{3}$. Similarly, the cohesive energy for hcp Ru is determined to be $7.154 \mathrm{eV}$ with the PAW method and $7.169 \mathrm{eV}$ with the FLAPW method, while the value given in Ref. 32 is $6.74 \mathrm{eV}$. In the PAW calculation, the ground electronic state for the free $\mathrm{Ru}$ is $[\mathrm{Kr}] 4 d^{7} 5 s^{1}$, with an energy which is $2.113 \mathrm{eV}$ lower than the reference state used for constructing the Ru PAW potential. For consistency, all of the following determination of cohesive energies refers to these free-atom ground-state calculations carried out with tetrahedron Brillion zone integration.

Next, we analyze the structural stability and magnetic moment of bulk V structures. Figure 1 shows that bec V has the lowest total energy, as expected, while hcp V has the highest one, and the energy difference is $0.26 \mathrm{eV} /$ atom. The minimal

TABLE I. Calculated bulk properties: lattice constant $(a)$ and cohesive energy $\left(E_{c}\right)$. Unless specified explicitly, results are obtained with the PAW method. The "hcp V", system is explained in Sec. III. $d_{n n}$ and $\Omega$ represent nearest-neighbor distance and atomic volume, respectively.

\begin{tabular}{ccccc}
\hline \hline Systems & $a(\AA)$ & $d_{n n}(\AA)$ & $\Omega\left(\AA^{3}\right)$ & $E_{c}(\mathrm{eV})$ \\
\hline bcc V & 3.00 & 2.60 & 2.79 & 5.399 \\
& & & & $5.600($ FLAPW $)$ \\
fcc V & 3.83 & 2.71 & 2.82 & 5.155 \\
hcp V & $2.60(\mathrm{c} / \mathrm{a}=1.82)$ & 2.60 & 2.82 & 5.144 \\
hcp V* & $2.74(\mathrm{c} / \mathrm{a}=1.65)$ & 2.74 & 2.87 & 5.091 \\
hcp $\mathrm{Ru}$ & $2.74(\mathrm{c} / \mathrm{a}=1.60)$ & 2.68 & 2.84 & 7.154 \\
& & & 7.169 (FLAPW) \\
\hline \hline
\end{tabular}



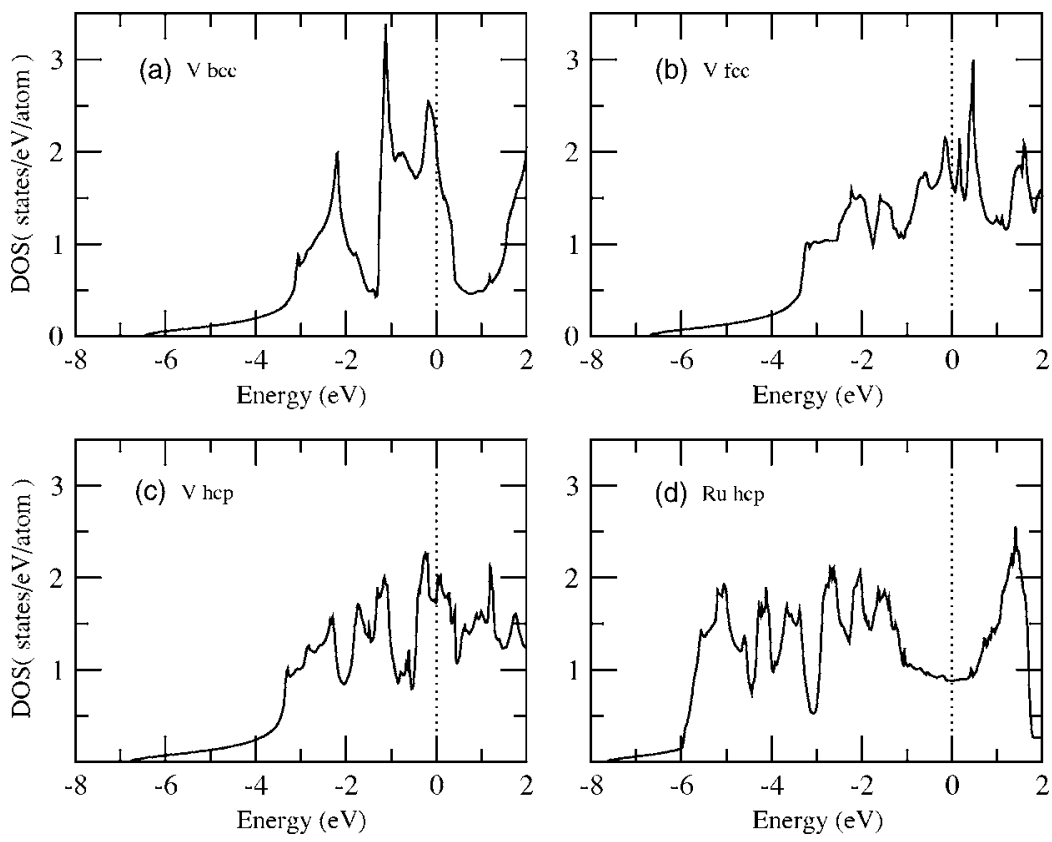

FIG. 2. The total density of states for bcc V (a), fcc V (b), hep V (c), and hep Ru (d). The dotted line at $0 \mathrm{eV}$ denotes the Fermi level. total energy of fcc V is slightly lower than that of the hep phase (by $0.02 \mathrm{eV} /$ atom). In Fig. 1, the variation of magnetic moment with the atomic volume $(\Omega)$ is also plotted. For bcc V [Fig. 1(a)], we can see clearly three magnetic phases (nonmagnetic, low-spin, and high-spin states) separated by two singularities at $\Omega=16.4$ and $25.9 \AA^{3} /$ atom (lattice constant $a=3.20$ and $3.73 \AA$ ), respectively. The calculated lattice constants of these two critical points are rather close to the values of 3.41 and $3.73 \AA$ given in Ref. 33, respectively. At the total energy minimal, $\Omega(a)$ is equal to $13.5 \AA^{3} /$ atom $(3.0 \AA)$, which falls in the nonmagnetic region. Upon expansion, this system would undergo complex phase transitions. ${ }^{33}$ It is clear that all three bulk structures are nonmagnetic at their respective equilibrium lattice constants as given in Table I. The fcc structure [Fig. 1(b)] shows similar characteristics with the two critical atomic volumes $\Omega$ being at 20.0 and $24.5 \AA^{3}$ /atom, respectively. Since the hcp structure has two independent lattice constants $a$ and $c$, the cell shape $(c / a)$ is not uniquely defined at a given volume. Therefore, we had to locate the minimum of the total energy as a function of $c / a$ for a given atomic volume, and the results are displayed in Fig. 1(c). Figure 1(c) indicates that the hcp structure also shows similar characteristics. Interestingly, the critical volume of $17.6 \AA^{3}$ /atom for the nonmagnetic- to low-spin-state transition is considerably smaller than that for the fcc structure, though the critical volumes for the low-spin to high-spin transition in both structures are nearly the same. The calculated magnetic moment fluctuates visibly especially in the low-spin region [Fig. 1(c)]. This is because the total energy has a very broad minimum as a function of $c / a$, and this makes it rather difficult to determine precisely the theoretical $c / a$ ratio. On the other hand, the magnetic moment is rather sensitive to the cell shape $(c / a)$. For bulk hcp $\mathrm{Ru}$, the structure with the lowest total energy is nonmagnetic as well and the variation of magnetic moment with lattice constant is similar to previous studies. ${ }^{29}$

The densities of states (DOS) for bcc V, fcc V, hcp V, and hcp $\mathrm{Ru}$ at their respective lowest-total-energy configuration are plotted in Fig. 2. The origin of the horizontal energy axis is shifted to the Fermi energy $\left(E_{f}\right)$ of each system. The DOS at the $E_{f}\left[n\left(E_{f}\right)\right]$ for each system is $2.07,1.65,1.73$, and 0.88 states/eV/atom, respectively. Using the exchangecorrelation integrals (I) calculated by $\mathrm{Janak}^{34}$ for bcc V $(0.35 \mathrm{eV})$ and fcc $\mathrm{Ru}(0.30 \mathrm{eV})$, all four structures give $n\left(E_{f}\right) I<1$. According to the Stoner criteria, ${ }^{35}$ these structures should be nonmagnetic, which is consistent with our spinpolarized calculations as given in Fig. 1.

\section{THIN-FILM PROPERTIES}

In this section, we report on calculations of the magnetic moments in thin films of a few monolayers of $\mathrm{V}$ on the $\mathrm{Ru}$ (0001) substrate. We assume that V layers have a pseudomorphic growth on the $\mathrm{Ru}$ substrate. All the in-plane lattice constants are assumed to be that of $\mathrm{Ru}$ in bulk hcp phase, which is $2.74 \AA$. Since the hcp V-V interlayer spacing $(1.38 \AA)$ perpendicular to $(0001)$ surface is close to the $\mathrm{Ru}-\mathrm{Ru}$ interlayer spacing (1.34 $\AA$ ), in our thin-film calculations, we use the $\mathrm{Ru}-\mathrm{Ru}$ interlayer spacing to construct all the thin-film structures, which is justified by surface relaxation results. The thin-film structures are simulated with the standard slab-supercell approach. The thickness of the whole unit cell for all thin-film structures considered in this paper is limited to 20 monolayers. The $\mathrm{Ru}$ bulk substrates are approximated by 7 monolayers. Thus, for the case of 1 monolayer of $\mathrm{V}$ on $\mathrm{Ru}(0001)$, the vacuum spacing is 11 monolayer thick; for the case of 3 monolayers of $\mathrm{V}$ on $\mathrm{Ru}$ (0001), the vacuum spacing is 7 monolayer thick.

One important question in considering these thin films is the following: During epitaxial layer-by-layer growth, should these atomic layers adopt bcc, fcc, or hcp stacking? According to previous experimental studies, ${ }^{6}$ during the growth of $\mathrm{Cr} / \mathrm{Ru}(0001)$ superlattices, $\mathrm{Cr}$ atomic layers first adopt the hcp structure of the $\mathrm{Ru}$ (0001) surface and switch completely to the bcc structure when $\mathrm{Cr}$ layers become thicker than three 


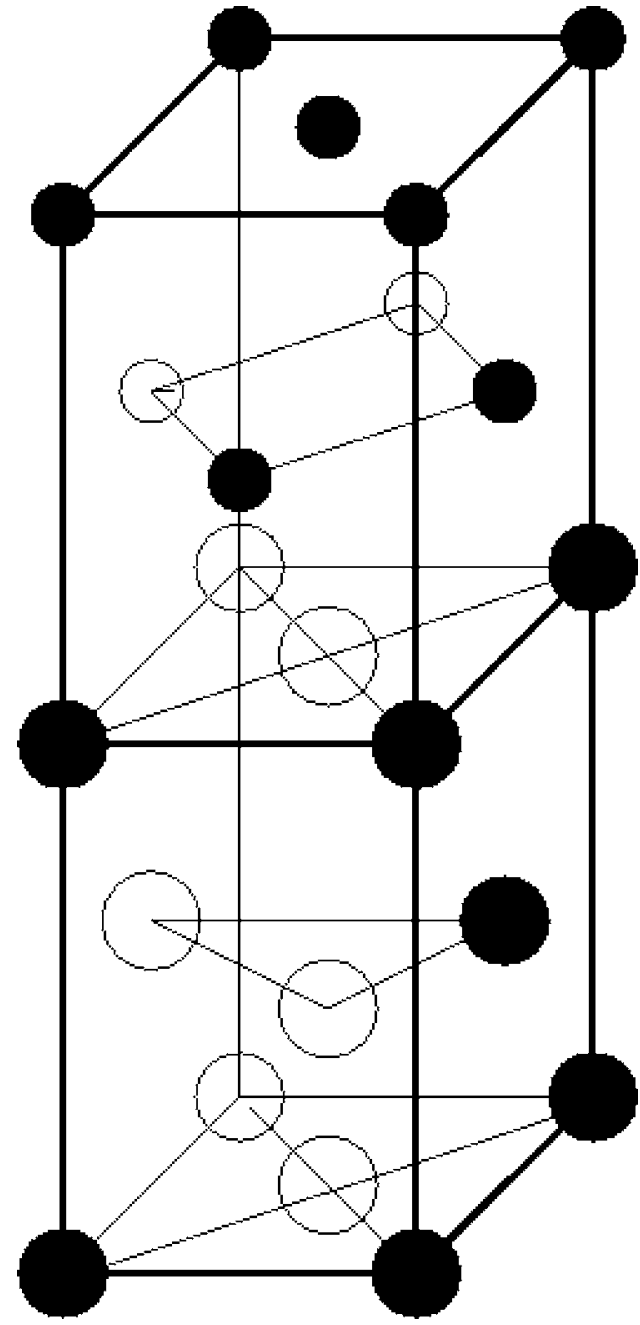

FIG. 3. A schematic picture showing the matching of $\mathrm{V}$ bcc (110) surfaces with Ru hcp (0001) surfaces. Large spheres denote the $\mathrm{Ru}$ atoms and small spheres represent the $\mathrm{V}$ atoms.

monolayers. ${ }^{6}$ Thus, we have calculated the following systems: $\mathrm{V}_{1}(\mathrm{hcp}) / \mathrm{Ru}, \mathrm{V}_{1}(\mathrm{fcc}) / \mathrm{Ru}, \mathrm{V}_{3}(\mathrm{hcp}) / \mathrm{Ru}, \mathrm{V}_{3}(\mathrm{fcc}) / \mathrm{Ru}$, and $\mathrm{V}_{3}(\mathrm{bcc}) / \mathrm{Ru}$, which refer to 1 or 3 monolayers of $\mathrm{V}$ atoms adopting hcp, fcc, or bcc stacking on Ru (0001) surfaces. The Brillouin zone is sampled with a $16 \times 16 \times 2$ grid which gives 60 or 147 irreducible $k$ points for hcp/fcc or bcc stacking, respectively. The structure of bcc stacking on hcp (0001) surface is called hp structure in previous studies. ${ }^{29} \mathrm{~A}$ schematic picture of this surface structure is given in Fig. 3. It is a strained match between the bcc (110) surface cell (defined by long edge [110] and short edge [100]) and hcp (0001) surface cell (defined by long edge [11 $\overline{2} 0]$ and short

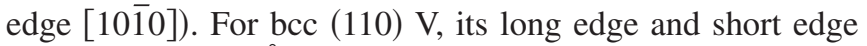
are 4.25 and $3.00 \AA$, respectively, which gives a surface area of $12.75 \AA^{2}$. For hcp (0001) Ru, its long edge and short edge are 4.74 and $2.74 \AA$, respectively, which gives a surface area of $12.98 \AA^{2}$. The calculation results are presented in Table II. Note that the cohesive energy as given in Table II for these thin films is obtained by substracting the total energy contribution of each individual atom in its free state.

The calculations for the unsupported $\mathrm{V}_{3}(\mathrm{hcp})$ thin film show that this system has a total magnetic moment of $2.3 \mu_{B}$.
TABLE II. Thin-film results calculated with the PAW method. With respect to the local magnetic moment $\left(m_{l}\right)$, the $\mathrm{V}$ atoms in the first row are exposed to the vacuum, the $\mathrm{V}$ atoms in the second row are located in the subsurface layer, and the $\mathrm{V}$ atoms in the third row are on the second subsurface layer. Since the Ru layers do not have an appreciable magnetic moment, only the Ru layer at the interface is reported. The cohesive energy $\left(E_{c}\right)$ and total magnetic moment $\left(m_{t}\right)$ include contributions from all atoms in the unit cell (no division of two is applied).

\begin{tabular}{lccr}
\hline \hline Systems & $E_{c}(\mathrm{eV})$ & $m_{t}(\mu \mathrm{B})$ & $m_{l}(\mu \mathrm{B})$ \\
\hline V3(hcp) & 13.428 & 2.40 & $\mathrm{~V} 0.92$ \\
$\begin{array}{l}\text { (unsupported) } \\
\text { V1(hcp)/Ru }\end{array}$ & 58.546 & 2.37 & $\mathrm{~V} 0.03$ \\
& & & $\mathrm{~V} 0.90$ \\
V1(fcc)/Ru & 58.407 & 1.79 & $\mathrm{Ru}-0.05$ \\
& & & $\mathrm{~V} 0.72$ \\
V3(hcp)/Ru & 79.238 & 0.00 & $\mathrm{Ru}-0.06$ \\
(relaxed) & & & 0.00 \\
V3(hcp)/Ru & 79.180 & 0.00 & 0.00 \\
V3(fcc)/Ru & 79.260 & 0.11 & $\mathrm{~V}-0.02$ \\
& & & $\mathrm{~V} 0.02$ \\
& & & $\mathrm{~V} 0.04$ \\
V3(bcc)/Ru & & & $\mathrm{Ru}-0.00$ \\
\hline \hline
\end{tabular}

The local magnetic moment for the outmost $\mathrm{V}$ atom is about $0.9 \mu_{B}$. The local magnetic moment for the $\mathrm{V}$ atom in the middle is zero. For the Ru (0001) substrate itself, a previous study ${ }^{29}$ has shown that a Ru monolayer has a local magnetic moment of about $0.3 \mu_{B}$ and there is no magnetic moment for two or four layers.

For one monolayer of $\mathrm{V}$ on $\mathrm{Ru}$, the occupation of hcp sites is more stable than the occupation of the fcc site by about $0.07 \mathrm{eV}$ per $\mathrm{V}$ atom. The local magnetic moment on $\mathrm{V}$ in $\mathrm{V}_{1}(\mathrm{hcp}) / \mathrm{Ru}$ and $\mathrm{V}_{1}(\mathrm{fcc}) / \mathrm{Ru}$ is 0.90 and $0.72 \mu_{B}$, respectively. The total magnetic moment per unit cell is $2.37 \mu_{B}$ and $1.79 \mu_{B}$ for $\mathrm{V}_{1}(\mathrm{hcp}) / \mathrm{Ru}$ and $\mathrm{V}_{1}(\mathrm{fcc}) / \mathrm{Ru}$, respectively. For three atomic layers of $\mathrm{V}$ on the $\mathrm{Ru}$ (0001) surface, the bcc stacking is the most stable. The cohesive energy per $\mathrm{V}$ atom is only $0.15 \mathrm{eV}$ larger than that in hcp stacking, in comparision with the bulk calculation in Sec. III that the bec V is $0.3 \mathrm{eV}$ more stable than hcp V. The total energy of the fcc stacking is close to the hcp stacking. The magnetic properties of these thicker film structures are quite different from the $\mathrm{V}_{1}$ (hcp)/Ru case. The total magnetic moment for the whole unit cell is zero for hcp and bcc stacking and only $0.11 \mu_{B}$ for fcc stacking. Here extreme care has been taken to make sure that the calculated moment is converged. The energy difference criterion is $10^{-8} \mathrm{eV}$.

In the case of $V_{3}(\mathrm{hcp}) / \mathrm{Ru}$, surface relaxation is performed to study its effect on the magnetic moment. As presented in Table II, the result indicates that the effect is very small and the magnetic property is almost the same. In this calculation the whole unit cell has 13 atoms and the positions of the innermost $3 \mathrm{Ru}$ atoms are fixed to resemble the bulk structure. The relaxation is carried out with spin polarization. 


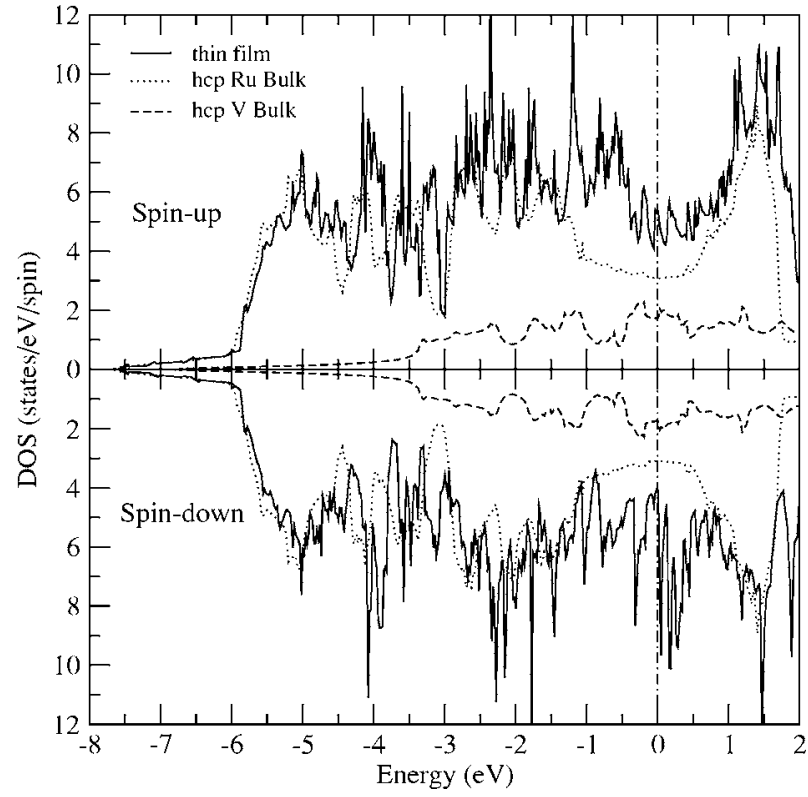

FIG. 4. Spin-polarized density of states (DOS) for one monolayer of $\mathrm{V}$ on the $\mathrm{Ru}$ (0001) surface (thin film), together with DOS of bulk hcp V and hcp Ru. Since each unit cell has seven Ru and two $\mathrm{V}$ atoms, bulk DOS for $\mathrm{Ru}$ and $\mathrm{V}$ are scaled up by a factor of 7 and 2 , respectively.

Force criterion is $0.01 \mathrm{eV} / \AA$. At the interface, the interlayer spacing $\mathrm{V}_{1}-\mathrm{Ru}_{1}$ is shrunk by about $3.3 \%$. Neighboring $\mathrm{V}_{1}$ $-\mathrm{V}_{2}$ is shrunk by about $0.3 \%$. The outmost $\mathrm{V}_{2}-\mathrm{V}_{3}$ is contracted by about $1.1 \%$. Neighboring $\mathrm{Ru}_{1}-\mathrm{Ru}_{2}$ is shrunk by about $0.1 \% . \mathrm{Ru}_{2}-\mathrm{Ru}_{3}$ is shrunk by about $2.5 \%$. The positions of the inner three Ru layers are frozen to resemble the bulk structure. Nevertheless, the net forces acting on them are still large $(0.25 \mathrm{eV} / \AA)$, which indicates that modeling of the $\mathrm{Ru}$ substrate with seven layers is not sufficient. An easier relaxation without considering spin polarization gives very similar results, i.e., $\mathrm{V}_{1}-\mathrm{Ru}_{1}$ by $-3.1 \%, \mathrm{~V}_{1}-\mathrm{V}_{2}$ by $-0.0 \%, \mathrm{~V}_{2}-\mathrm{V}_{3}$ by $-0.5 \%, \mathrm{Ru}_{1}-\mathrm{Ru}_{2}$ by $-0.0 \%$, and $\mathrm{Ru}_{2}-\mathrm{Ru}_{3}$ by $-3.0 \%$.

In Fig. 4, the spin-polarized DOS of $\mathrm{V}_{1}$ (hcp)/Ru is plotted. To see the surface and interface effects, bulk hcp Ru and hcp V spin-up DOS are also plotted with uniform scaling to account for the numbers of $\mathrm{Ru}$ and $\mathrm{V}$ atoms in the doubleside slab cell, respectively. For the spin-up DOS, there are occupied surface states at about $0.6 \mathrm{eV}$ below the Fermi energy. For the spin-down DOS, these surface states are pushed up and centered at $0.30 \mathrm{eV}$ above the Fermi energy. At about -1.2 and $-2.3 \mathrm{eV}$ for spin-up and $-1.8 \mathrm{eV}$ for spin-down DOS, the DOS indicate the formation of strongly bound states. In Fig. 5 the spin-up DOS of three monolayers of V on Ru hcp(0001) surface is plotted. The spin-down DOS is similar to the corresponding spin-up DOS and thus is not plotted. In the energy window within about $3 \mathrm{eV}$ below the Fermi level and also $1 \mathrm{eV}$ above, the change of DOS with lattice stacking is visible (Fig. 5).

\section{SUPERLATTICE PROPERTIES}

In this section we report on the calculaton of properties for superlattices with $\mathrm{V}$ monolayers varying from 1 to 5 .

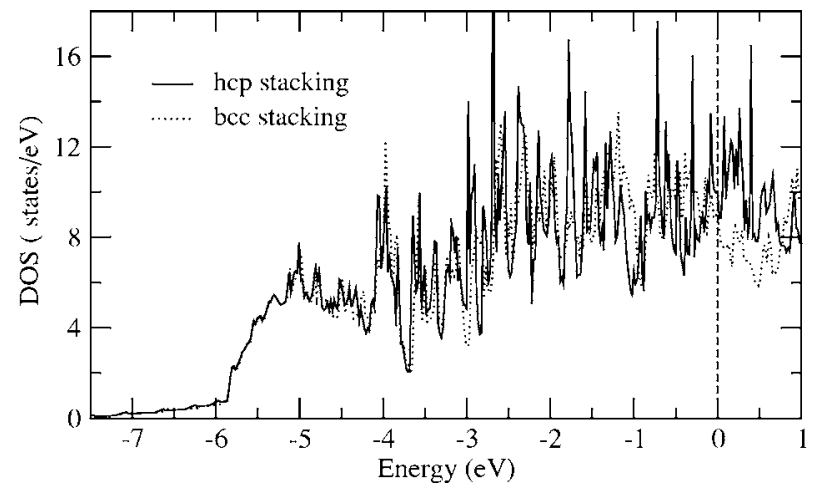

FIG. 5. Spin-polarized density of states (DOS) for three layers of $\mathrm{V}$ on $\mathrm{Ru}$ (0001). Only the spin-up DOS for hep stacking and bec stacking are given, because the profiles for spin-down DOS are essentially the same.

Because of symmetry constraints, in our construction of supercells the thickness of the Ru layer varies with the thickness of the $\mathrm{V}$ layer. Unless specified otherwise, $\mathrm{V}_{n} \mathrm{Ru}_{m}$ represents a superlattice of $n$ monolayers of hcp (0001) V and $m$ monolayers of hcp (0001) Ru stacked together. Superlattices composed of $n \mathrm{~V}$ monolayers stacked in fcc or bcc sequence are termed as $\mathrm{V}_{n}(\mathrm{fcc}) \mathrm{Ru}_{m}$ or $\mathrm{V}_{n}(\mathrm{bcc}) \mathrm{Ru}_{m}$. Calculations are performed for the following systems: $\mathrm{V}_{1} \mathrm{Ru}_{5}, \mathrm{~V}_{3} \mathrm{Ru}_{5}$, $\mathrm{V}_{3}(\mathrm{fcc}) \mathrm{Ru}_{5}, \mathrm{~V}_{3}(\mathrm{bcc}) \mathrm{Ru}_{5}, \mathrm{~V}_{5} \mathrm{Ru}_{5}, \mathrm{~V}_{2} \mathrm{Ru}_{6}$, and $\mathrm{V}_{4} \mathrm{Ru}_{6}$. Initially, we planned to do all the calculations with the PAW method. However, during our calculations of a few superlattice structures, we encountered quite a few numerical instabilities and it was not clear whether we could ignore them. Thus, we carried out systematic FLAPW calculations to cross-check the PAW results. In particular, $\mathrm{V}_{1} \mathrm{Ru}_{5}, \mathrm{~V}_{3} \mathrm{Ru}_{5}$, and $\mathrm{V}_{5} \mathrm{Ru}_{5}$ are studied with both the PAW and FLAPW methods. $\mathrm{V}_{3}(\mathrm{fcc}) \mathrm{Ru}_{5}$ and $\mathrm{V}_{3}(\mathrm{bcc}) \mathrm{Ru}_{5}$ are studied with the PAW method. $\mathrm{V}_{2} \mathrm{Ru}_{6}$ and $\mathrm{V}_{4} \mathrm{Ru}_{6}$ are studied with the FLAPW method. For the FLAPW calculations, the number of $k$ points in the IBZW is $114,80,140,88$, and 154 , respectively, for the $\mathrm{V}$ layer thickness varying from 1 to 5 monolayers. The symmetry of superlattices with an even ( 2 or 4$)$ number of $\mathrm{V}$ monolayers is higher than that with an odd (1, $3,5)$ number of $\mathrm{V}$ monolayers. For the PAW calculations, the number of $k$ points is 150 for $\mathrm{V}_{n} \mathrm{Ru}_{m}$ where $n=1,3,5$. For $\mathrm{V}_{3}(\mathrm{fcc}) \mathrm{Ru}_{5}$ and $\mathrm{V}_{3}(\mathrm{bcc}) \mathrm{Ru}_{5}$, the number of $k$ points is 213 and 365, respectively. The results are given in Table III. Here we note that by substracting the free-atom ground-state energies calculated with the methods described in Sec. III, the cohesive energies obtained by the FLAPW and PAW methods are comparable.

Without considering lattice relaxation, our calculations indicate that $\mathrm{V}_{1} \mathrm{Ru}_{5}, \mathrm{~V}_{4} \mathrm{Ru}_{6}$, and $\mathrm{V}_{5} \mathrm{Ru}_{5}$ are nonmagnetic whilst $\mathrm{V}_{2} \mathrm{Ru}_{6}$ and $\mathrm{V}_{3} \mathrm{Ru}_{5}$ have small magnetic moments. For $\mathrm{V}_{2} \mathrm{Ru}_{6}$, the FLAPW calculation indicated that the whole unit cell has a magnetic moment of $0.45 \mu_{B}$. The local magnetic moment for $\mathrm{V}$ atom is about $0.22 \mu_{B}$ and the interface $\mathrm{Ru}$ becomes slightly antiferromagnetic with a local magnetic moment about $-0.02 \mu_{B}$. The magnetic moment in the interstitial region is about $0.07 \mu_{B}$. For $\mathrm{V}_{3} \mathrm{Ru}_{5}$, the FLAPW calculations with initial atomic spin configuration either ferromagntic (FM) or antiferromagnetic (AFM) give the same 
TABLE III. Cohesive energy $\left(E_{c}\right)$ and total $\left(m_{t}\right)$ and local $\left(m_{l}\right)$ magnetic moments in the superlattice calculated with the PAW $(\dagger)$ and FLAPW ( $\ddagger$ methods. The $\mathrm{V}$ atoms are listed in the order of their sequential stacking in the unit cell. Since the Ru layers do not have appreciable magnetic moment, only the $\mathrm{Ru}$ layer at the interface is reported.

\begin{tabular}{lccr}
\hline \hline Systems & $E_{c}(\mathrm{eV})$ & $m_{t}(\mu \mathrm{B})$ & $m_{l}(\mu \mathrm{B})$ \\
\hline V1Ru5 $^{\ddagger}$ & 41.240 & 0.00 & 0.00 \\
V1Ru5 $^{\dagger}$ & 40.581 & 0.00 & 0.00 \\
V2Ru6 $^{\ddagger}$ & 53.488 & 0.45 & $\mathrm{~V} 0.22$ \\
& & & $\mathrm{Ru}-0.02$ \\
V2Ru6 $^{\ddagger}$ & 53.577 & 0.04 & $\mathrm{~V} 0.03$ \\
(relaxed) $^{\text {V3Ru5 }}{ }^{\dagger}$ & & & $\mathrm{Ru}-0.00$ \\
& 51.465 & 0.25 & $\mathrm{~V} 0.03$ \\
& & & $\mathrm{~V} 0.09$ \\
V3Ru5 $^{\ddagger}$ & & & $\mathrm{Ru}-0.00$ \\
& 51.369 & 0.26 & $\mathrm{~V} 0.03$ \\
& & & $\mathrm{~V} 0.09$ \\
V3Ru5 (relaxed) $^{\dagger}$ & 51.533 & 0.00 & $\mathrm{Ru} 0.00$ \\
V3(fcc)Ru5 $^{\dagger}$ & 51.488 & 0.00 & 0.00 \\
V3(bcc)Ru5 $^{\dagger}$ & 51.893 & 0.00 & 0.00 \\
V4Ru6 $^{\ddagger}$ & 64.586 & 0.00 & 0.00 \\
V5Ru5 $^{\ddagger}$ & 62.719 & 0.00 & 0.00 \\
V5Ru5 $^{\dagger}$ & 61.062 & 0.00 & 0.00 \\
\hline \hline
\end{tabular}

results: the whole unit cell has a magnetic moment of $0.26 \mu_{B}$; the local magnetic moment for $\mathrm{V}$ atom at the $\mathrm{V}-\mathrm{Ru}$ interface is $0.09 \mu_{B}$ and the $\mathrm{V}$ atom in the middle $(\mathrm{V}-\mathrm{V}-\mathrm{V})$ is $0.03 \mu_{B}$; the magnetic moment in the interstitial region is about $0.04 \mu_{B}$. For a calculation initialized with the AFM spin configuration, it is easy to observe that during the self-consistent cycles the magnetic moment distribution makes the transition toward the FM calculation and very good convergence can be obtained. The PAW calculation agrees very well with the above calculation. The total magnetic moment for the whole unit cell is $0.25 \mu_{B}$ and the total cohesive energy is about $0.1 \mathrm{eV}$ larger.

For the superlattice of three monolayers of $\mathrm{V}$, the effect of stacking sequence has been studied. As given in Table III, the PAW result indicates that both fcc and bcc stacking are more stable than hcp stacking. However, the cohesive energy for $\mathrm{V}_{3}$ (fcc) $\mathrm{Ru}_{5}$ and $\mathrm{V}_{3}(\mathrm{bcc}) \mathrm{Ru}_{5}$ is larger than $\mathrm{V}_{3} \mathrm{Ru}_{5}$ by only 0.02 and $0.07 \mathrm{eV}$ per $\mathrm{V}$ atom, respectively. Note that the cohesive energy for bulk bcc $\mathrm{V}$ is larger than for bulk hcp V by $0.3 \mathrm{eV}$ per $\mathrm{V}$ atom. This suggests the possibility of stabilizing ultrathin hep $\mathrm{V}$ films in $\mathrm{V} / \mathrm{Ru}$ superlattices, as indicated in recent experiments. ${ }^{18}$ The change of the magnetic property is also noticeable. For both $\mathrm{V}_{3}(\mathrm{fcc}) \mathrm{Ru}_{5}$ and $\mathrm{V}_{3}$ (bcc) $\mathrm{Ru}_{5}$, the total magnetic moment of the whole cell is close to zero and each $\mathrm{V}$ atom becomes nonmagnetic as well.

In Fig. 6, the total (spin-up plus spin-down) DOS of $\mathrm{V}_{3}$ (bcc) $\mathrm{Ru}_{5}$ is plotted. The DOS of bulk bcc $\mathrm{V}$ and hcp $\mathrm{Ru}$ scaled by their corresponding numbers of atoms in $\mathrm{V}_{3}$ (bcc) $\mathrm{Ru}_{5}$ are also plotted. In reference to their respective

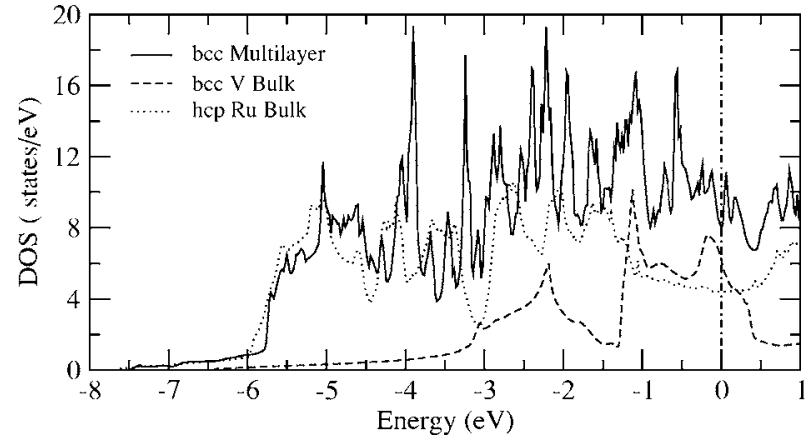

FIG. 6. Total density of states (DOS) for the $\mathrm{V}_{3}(\mathrm{bcc}) \mathrm{Ru}_{5}$ superlattice, together with bulk bcc V and hep Ru. As each unit cell has five $\mathrm{Ru}$ and three $\mathrm{V}$ atoms, the bulk DOS for Ru and $\mathrm{V}$ are scaled up by a factor of 5 and 3 , respectively.

bulk DOS, we notice that in the multilayer structure, the $\mathrm{Ru}$ contribution to DOS moves up in energy towards the Fermi level and that the $\mathrm{V}$ contribution to DOS moves down in energy away from the Fermi level. This suggests that $\mathrm{V}$ atoms gain stability by forming multilayer structure with $\mathrm{Ru}$ atoms while $\mathrm{Ru}$ atoms lose stability by forming multilayer structure with $\mathrm{V}$ atoms, consistent with the change of cohesive energy as given in Table III. The sharp peak at about $3.9 \mathrm{eV}$ below the Fermi level indicates the formation of interface states. Other peaks are readily identifiable from their bulk contributions. In Fig. 7, the spin-polarized DOS of the $\mathrm{V}_{3} \mathrm{Ru}_{5}$ are presented, together with the spin-polarized DOS of $\mathrm{V}_{3}(\mathrm{bcc}) \mathrm{Ru}_{5}$ for comparison. At the lower edge of the band, we can see that the DOS is almost the same, including interface states at about $-3.9 \mathrm{eV}$. At higher energy, the effect of stacking order becomes visible.

An essential purpose of this study is to determine under which conditions a stable magnetic moment can exist in $\mathrm{V}_{n} \mathrm{Ru}_{m}$ multilayers. Thus, it is interesting to find that $\mathrm{V}_{2} \mathrm{Ru}_{6}$

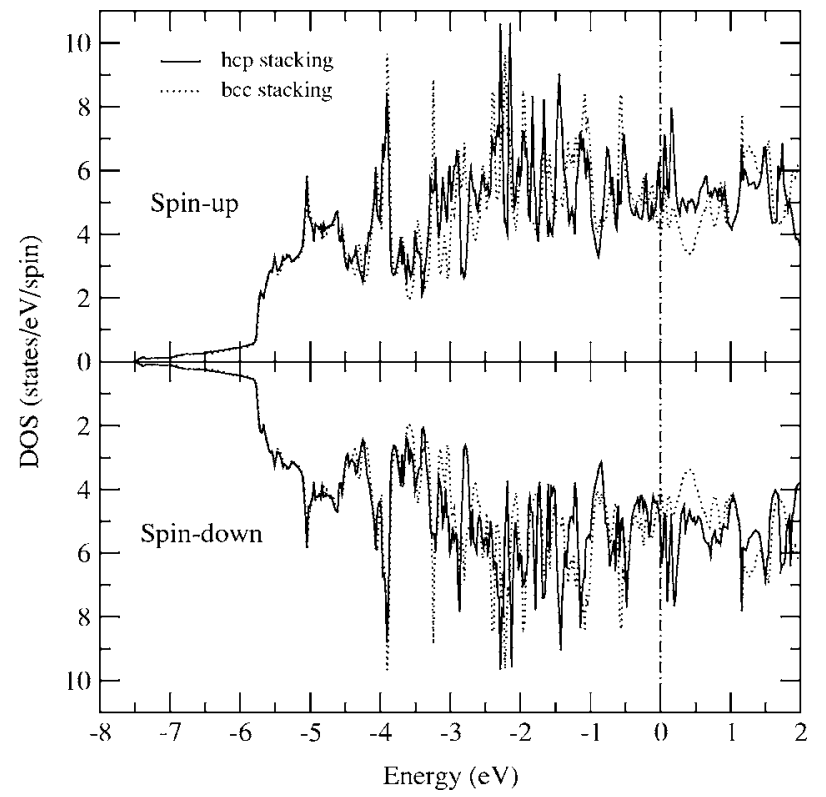

FIG. 7. Spin-polarized density of states (DOS) for $\mathrm{V}_{3}(\mathrm{hcp}) \mathrm{Ru}_{5}$ and $\mathrm{V}_{3}(\mathrm{bcc}) \mathrm{Ru}_{5}$ multilayer structures. 
and $\mathrm{V}_{3} \mathrm{Ru}_{5}$ have stable magnetic moments, though all the others do not. Since it is unlikely that lattice relaxation would turn nonmagnetic systems into magnetic ones, here we single out $\mathrm{V}_{2} \mathrm{Ru}_{6}$ and $\mathrm{V}_{3} \mathrm{Ru}_{5}$ to consider the effects of lattice relaxation on the magnetic moments. For $\mathrm{V}_{2} \mathrm{Ru}_{6}$, we carried out lattice relaxation with the FLAPW method. The optimized structure is obtained by having the interlayer spacing of the whole unit cell uniformly shrunk by about $1.86 \%$ and then carrying out quasi-Newton dynamics to minimize the force acting on each atom. The relaxation energy is about $0.09 \mathrm{eV}$. The $\mathrm{V}-\mathrm{V}$ interlayer spacing is expanded by about $1.4 \%$. The interface $\mathrm{V}-\mathrm{Ru}$ spacing is shrunk by about $1.4 \%$. The $\mathrm{Ru}-\mathrm{Ru}$ spacing close to the interface is expanded by about $1 \%$. The residual force with Pulay correction is less than $0.4 \mathrm{mRy} / \mathrm{a}$.u. The total magnetic moment is decreased to $0.04 \mu_{B}$ and the local magnetic moment of $\mathrm{V}$ atoms is about $0.03 \mu_{B}$ (see Table III).

For $\mathrm{V}_{3} \mathrm{Ru}_{5}$, there is no inversion symmetry and the convergence is slow with the FLAPW method. Thus, we report on a relaxation study of this structure with the PAW method. The relaxation with the PAW calculation is a full lattice structure relaxation including in-plane lattice constant change while maintaining the symmetry. The optimized structure has an in-plane lattice constant shrunk by about $0.2 \%(0.006 \AA)$ and out-of-plane lattice constant shrunk by about $1.6 \%(0.274 \AA)$. If we assume that the total energy is a uniform function of volume and translate the $0.2 \%$ in-plane change to out-of-plane change, in total the out-of-plane lattice constant contraction would be $2.0 \%(0.354 \AA)$. The force criterion used is $0.01 \mathrm{eV} / \AA$, which is about $0.4 \mathrm{mRy} / \mathrm{a} . \mathrm{u}$., and the same as the criterion used in the FLAPW calculation for $\mathrm{V}_{2} \mathrm{Ru}_{6}$. The interlayer spacing change is as follows: $\mathrm{V}-\mathrm{V}$ by $+0.1 \%(0.002 \AA), \mathrm{V}-\mathrm{Ru} 1$ by $-4.45 \%(-0.097 \AA), \mathrm{Ru} 1-\mathrm{Ru} 2$ by $+0.48 \%$ (0.010 $)$, and Ru2-Ru3 - $2.40 \%(0.053 \AA)$. Once again, $V$ and Ru atoms in the interface tend to move closer and in their neighboring layers away from the interface tend to move away. The relaxation energy of this structure is $0.068 \mathrm{eV}$ as determined from the PAW calculation. The disappearance of the small magnetic moment after relaxation is understandable. It is generally known that the magnetic moment will decrease upon the contraction of volumes. For the structures that we have studied here, relaxation leads to a reduction of their volume and the electrons become more densely packed. In Fig. 8, we show the effect of relaxation on the DOS in $\mathrm{V}_{3} \mathrm{Ru}_{5}$. In the low-energy edge of the valence band at about $-5.8 \mathrm{eV}$, it is clear that after lattice relaxation electron states are relocated to lower energy. For both spin-up and spindown directions, the interface states at about $-3.9 \mathrm{eV}$ shift to a lower energy and become broader. For both spin orientations, the original branched interface states at about $-3.7 \mathrm{eV}$ essentially merge into a single peak, reflecting the shrinking of the $\mathrm{V}-\mathrm{Ru}$ interlayer spacing after relaxation.

\section{CONCLUSIONS}

In summary, we have performed systematic firstprinciples density-functional studies of the electronic and magnetic properties of ultrathin films of $\mathrm{V}$ on $\mathrm{Ru}(0001)$ and

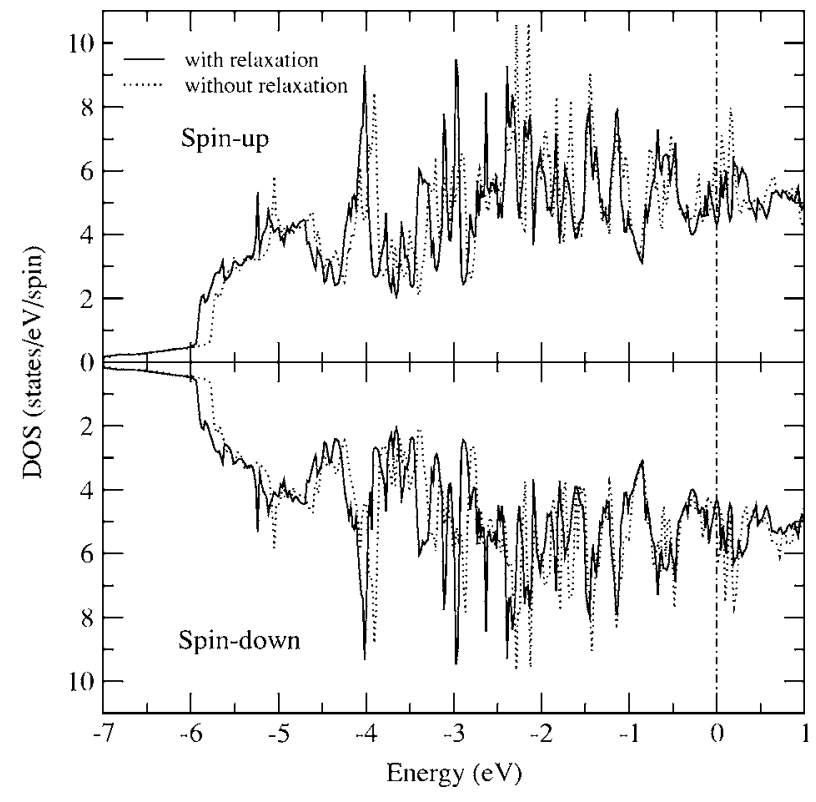

FIG. 8. Comparison of spin-polarized density of states (DOS) for $\mathrm{V}_{3} \mathrm{Ru}_{5}$ with (solid line) and without (dotted line) lattice relaxation.

also $\mathrm{V} / \mathrm{Ru}$ superlattices as well as bulk $\mathrm{V}$ in bcc, fcc, and hcp structures. We find that the three bulk structures are nonmagnetic at their respective minimal-energy lattice constants. However, all three structures would transit first to a low-spin ferromagnetic state and then to a high-spin state as the lattices expand. We also find that thin films with one monolayer of $\mathrm{V}$ in either hcp or fcc stacking sequence on $\mathrm{Ru}(0001)$ have a magnetic moment in the order of $1 \mu_{B}$. A thin film of three monolayers of $\mathrm{V}$ in fcc stacking sequence is weakly ferromagnetic, though the other thin films with more than one monolayer of $\mathrm{V}$ are essentially nonmagnetic. Our calculations show that the $\mathrm{V}_{2}(\mathrm{hcp}) / \mathrm{Ru}_{6}(\mathrm{hcp})$ and $\mathrm{V}_{3}(\mathrm{hcp}) / \mathrm{Ru}_{5}(\mathrm{hcp})$ superlattices exhibit ferromagnetism with a small total magnetic moment of a few tenths of $\mu_{B}$. Lattice relaxation has the trend of decreasing the magnitude of the magnetic moments. All other $\mathrm{V}_{n} / \mathrm{Ru}_{m}(\mathrm{hcp})$ superlattices ( $n=1,4,5$ and $m=5,6$ as well as $n=3$ with fcc or bcc stacking sequence) are essentially nonmagnetic. A free-standing film of three $\mathrm{V}$ monolayers in hcp stacking sequence is ferromagnetic with sizable magnetic moments. Finally, our calculations indicate that the stacking sequence is important for the energetic stability and also has significant effects on the formation of stable atomic magnetic moments of $\mathrm{V}$ in these systems.

\section{ACKNOWLEDGMENTS}

The authors thank M. Albrecht for discussing his experimental results on $\mathrm{V} / \mathrm{Ru}$ superlattices and also for his helpful comments on the manuscript. The authors also acknowledge financial support from National Science Council and NCTS/ TPE of ROC as well as computing time from National Center for High-Performance Computing of ROC. 
*Electronic address: tjzhang@quantumstates.com; URL: http:// www.quantumstates.com/tjzhang

†Electronic address: gyguo@phys.ntu.edu.tw

${ }^{1}$ W. Keune, R. Holbauer, U. Gonser, J. Lauer, and D. L. Williamson, J. Appl. Phys. 48, 2976 (1977).

${ }^{2}$ G. A. Prinz, Phys. Rev. Lett. 54, 1051 (1985).

${ }^{3}$ B. Heinrich, A. S. Arrott, J. F. Cochran, C. Liu, and K. Myrtle, J. Vac. Sci. Technol. A 64, 1376 (1986).

${ }^{4}$ Tianjiao Zhang, Ph.D. thesis, University of Tennessee, 2003.

${ }^{5}$ B. Schulz and K. Baberschke, Phys. Rev. B 50, 13467 (1994).

${ }^{6}$ M. Albrecht, M. Maret, J. Köhler, B. Gilles, R. Poinsot, J. L. Hazemann, J. M. Tonnerre, C. Teodorescu, and E. Bucher, Phys. Rev. Lett. 85, 5344 (2000)

${ }^{7}$ S. B. Zhang, Lixin Zhang, Lifang Xu, E. G. Wang, Xi Liu, JinFeng Jia, and Qi-Kun Xue, Phys. Rev. B 69, 121308(R) (2004).

${ }^{8}$ A. V. Ponomareva, L. V. Pourovskii, E. I. Isaev, Y. Kh. Vekilov, B. Johansson, and I. A. Abrikosov, Phys. Rev. B 68, 064409 (2003).

${ }^{9}$ Ilja Turek, Stefan Blügel, and Josef Kudrnovský, Phys. Rev. B 57, R11065 (1998).

${ }^{10}$ Houqian Sun, You-Hua Luo, Jijun Zhao, and Guanghou Wang, Phys. Status Solidi B 215, 1127 (1999).

${ }^{11}$ A. Rubio-Ponce, A. E. Garcia, and R. Baquero, Rev. Mex. Fis. 49, 411 (2003).

${ }^{12}$ I. G. Batyrev, J.-H. Cho, and L. Kleinman, Phys. Rev. B 63, 172420 (2001).

${ }^{13}$ G. Kresse, W. Bergermayer, and R. Podloucky, Phys. Rev. B 66, 146401 (2002).

${ }^{14}$ D. Spisák and J. Hafner, Phys. Rev. B 61, 4160 (2000).

${ }^{15}$ M. M. Schwickert, G. Y. Guo, M. A. Tomaz, W. L. O'Brien, and G. R. Harp, Phys. Rev. B 58, R4289 (1998).

${ }^{16}$ M. Kralj, P. Pervan, M. Milun, T. Valla, P. D. Johnson, and D. P. Woodruff, Phys. Rev. B 68, 245413 (2003); M. Kralj, P. Pervan, M. Milun, P. Lazic, Z. Crljen, R. Brako, J. Schneider, A. Rosenhahn, and K. Wandelt, Phys. Rev. B 68, 195402 (2003).

${ }^{17}$ G. D. Waddill, D. P. Moore, and O. Ozturk, Phys. Rev. B 61, 7301 (2000).
${ }^{18} \mathrm{M}$. Albrecht (private communication).

${ }^{19}$ P. E. Blöchl, Phys. Rev. B 50, 17953 (1994).

${ }^{20}$ P. Blaha, K. Schwarz, G. K. H. Madsen, D. Kvasnicka, and J. Luitz, Computer code WIEN2K, an augmented plane wave + local orbitals program for calculating crystal properties, Karlheinz Schwarz, Technical Universit ä Wien, Austria, 2001.

${ }^{21}$ G. Kresse and J. Furthmüller, Comput. Mater. Sci. 6, 15 (1996).

${ }^{22}$ C. S. Wang, B. M. Klein, and H. Krakauer, Phys. Rev. Lett. 54, 1852 (1985).

${ }^{23}$ J. Chen, D. Singh, and H. Krakauer, Phys. Rev. B 38, 12834 (1988).

${ }^{24}$ G. Y. Guo, H. Ebert, W. M. Temmerman, K. Schwarz, and P. Blaha, Solid State Commun. 79, 121 (1991).

${ }^{25}$ D. J. Singh, W. E. Pickett, and H. Krakauer, Phys. Rev. B 43, 11628 (1991).

${ }^{26}$ J. P. Perdew, K. Burke, and M. Ernzerhof, Phys. Rev. Lett. 77, 3865 (1996).

${ }^{27}$ G. Y. Guo and H. H. Wang, Chin. J. Phys. (Taipei) 38, 949 (2000).

${ }^{28}$ P. E. Blöchl, O. Jepsen, and O. K. Andersen, Phys. Rev. B 49, 16223 (1994).

${ }^{29}$ D. Spisák, R. Lorenz, and J. Hafner, Phys. Rev. B 63, 094424 (2001).

${ }^{30}$ G. Y. Guo, in Magnetic and Electronic Films-Microstructure, Texture and Applications to Data Storage, edited by P. W. DeHaven, D. P. Field, S. D. Harkness IV, J. A. Sutliff, J.A. Szpunar, L. Tang, T. Thomson, and M. D. Vaudin, MRS Symposia Proceedings No. 721 (Materials Research Society, Pittsburgh, 2002).

${ }^{31}$ S. H. Vosko, L. Wilk, and M. Nusair, Can. J. Phys. 58, 1200 (1980).

${ }^{32}$ C. Kittel, Introduction to Solid State Physics, 7th ed. (Wiley, New York, 1996).

${ }^{33}$ V. L. Moruzzi, P. M. Marcus, and P. C. Pattnaik, Phys. Rev. B 37, 8003 (1988).

${ }^{34}$ J. F. Janak, Phys. Rev. B 16, 255 (1977).

${ }^{35}$ E. C. Stoner, Proc. R. Soc. London, Ser. A 169, 339 (1939). 